\title{
Hyperspectral imaging for diagnosis and quality control in agri-food and industrial sectors
}

\author{
P. Beatriz Garcia-Allende, Olga M. Conde, Jesus Mirapeix, Adolfo Cobo and Jose M. \\ Lopez-Higuera \\ Photonics Engineering Group, University of Cantabria, Avda. Los Castros s/n, 39005 \\ Santander, Spain;
}

\begin{abstract}
Optical spectroscopy has been utilized in various fields of science, industry and medicine, since each substance is discernible from all others by its spectral properties. However, optical spectroscopy traditionally generates information on the bulk properties of the whole sample, and mainly in the agri-food industry some product properties result from the heterogeneity in its composition. This monitoring is considerably more challenging and can be successfully achieved by the so-called hyperspectral imaging technology, which allows the simultaneous determination of the optical spectrum and the spatial location of an object in a surface. In addition, it is a nonintrusive and non-contact technique which gives rise to a great potential for industrial applications and it does not require any particular preparation of the samples, which is a primary concern in food monitoring.

This work illustrates an overview of approaches based on this technology to address different problems in agri-food and industrial sectors. The hyperspectral system was originally designed and tested for raw material on-line discrimination, which is a key factor in the input stages of many industrial sectors. The combination of the acquisition of the spectral information across transversal lines while materials are being transported on a conveyor belt, and appropriate image analyses have been successfully validated in the tobacco industry. Lastly, the use of imaging spectroscopy applied to online welding quality monitoring is discussed and compared with traditional spectroscopic approaches in this regard.
\end{abstract}

Keywords: optical spectroscopy, hyperspectral imaging, non-destructive evaluation, food analysis, material classification, arc-welding monitoring

\section{INTRODUCTION}

Optical Spectroscopy (OS) covers all types of qualitative and quantitative analytical methods that are based on the interaction of light with living and non-living matter. ${ }^{1}$ For more than 200 years it has been widely utilized in various fields of science, industry and medicine. OS is highly specific since each substance is discernible from all others by its spectral properties. In addition, the requirements of the samples are not particularly restrictive. Measurement of various optical parameters as a function of wavelength/energy ("spectrum") provide valuable insights that are not, or not readily, attainable by other analytical methods. But traditional OS techniques generate information on the bulk properties of a sample or over a portion taken from it. ${ }^{2}$ However, in applications in the agri-food industry, for example, many aspects of ingredient and product properties result

Further author information: (Send correspondence to P.B.G.)

P.B.G.: E-mail: garciapb@unican.es; Telephone: +34 9422008 77; http://gif.teisa.unican.es/

Optical Sensing and Detection, edited by Francis Berghmans, Anna Grazia Mignani, Chris A. van Hoof, Proc. of SPIE Vol. 7726, 77260E - (C) 2010 SPIE · CCC code: 0277-786X/10/\$18 - doi: 10.1117/12.854506 

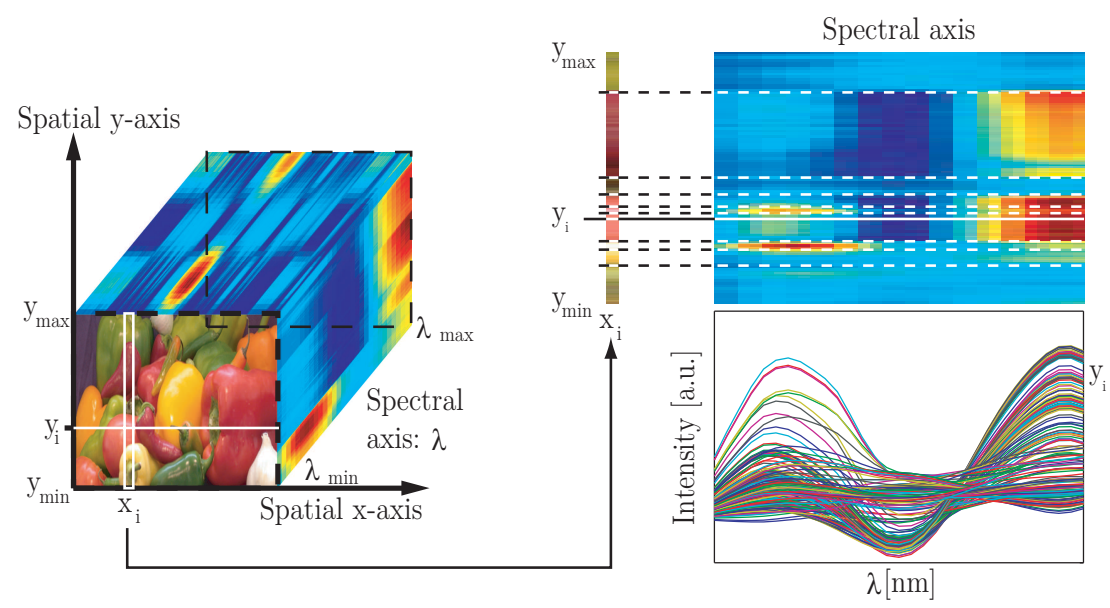

Figure 1. The concept of imaging spectroscopy is shown with a spectrum measured for each spatial element in an image.

from composition heterogeneity. The monitoring of this aspect of quality is considerably more challenging, and it can be successfully performed using imaging spectroscopy. The latter allows the simultaneous determination of the optical spectrum components of a particular interaction phenomenon between light and matter (absorption, scattering, etc.) and the spatial location of this interaction over a surface. This concept is shown in Figure 1. The acquired data will be a three-dimensional data set, the so-called datacube, defined by spatial $x$ and $y$ and wavelength $\lambda$ coordinates. ${ }^{3}$ Fig. 1 depicts the datacube consisting of the light reflected by the image shown at the foremost part of the cube. Spectral images and reflectance spectra of the vertical line of the image located in $x_{i}$ are presented on the right of Fig. 1, where the discontinuous lines highlight the correlation between the real and spectral images of the segment. A color gradation scale is employed to represent the spectra (approximately in the visible range) of each spatial position $y_{i}$ of the vertical lines. When the color of a pixel is close to red, it means that the intensity of the reflected radiation at that particular wavelength is high. On the contrary, if the pixel is blue, then it means that the intensity is small.

The systems that provide this kind of data are called imaging spectrometers and they are classified from the point of view of their data-acquisition modes. The most convencional types are shown in Figure 2. Non-imaging spectrometers are called whiskbroom scanners when they work as imaging spectrometers, because they provide the spectrum of a single point within the image, i.e. a column within the image cube. The recording of the whole image implies a double spatial scanning step, and, either scanning mechanics or several single point instruments are, therefore, required. These approaches are respectively time-consuming and expensive. Staring imagers, as filtered cameras, electronically scan the spectral domain, which means that the image is collected one spectral plane after the other. Although easy to implement by the employment of tunable filters, this configuration has a major drawback. The spectra for each pixel are not taken simultaneously, and, consequently, pixel-to-pixel spectral mixing could occur if the scene is moving while the spectra are being scanned. ${ }^{3}$ As an alternative to the filter configuration, tunability of the light source could also provide this spectral plane detection. If light specifications are related with material discrimination in terms of wavelength selection, ${ }^{4}$ the behaviour of the system could be improved. Finally, pushbroom scanner type of imaging spectrometers employ a detector in array to simultaneously scan the spectral dimension and one spatial dimension. Unlike color cameras that collect information in the three red, green, and blue bands or multispectral imaging achieved by tunable filters, this acquisition mode allows data collection in hundreds or thousands of spectral bands (hyperspectral) to produce 


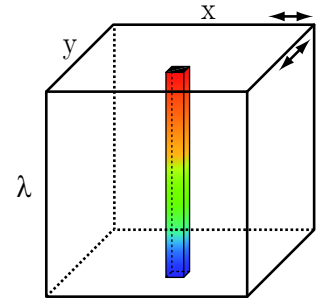

Whiskbroom spectrometer

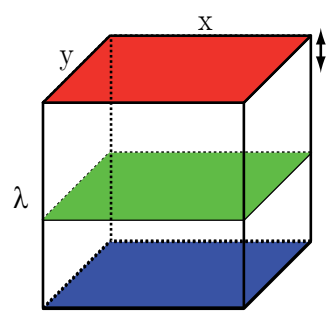

Filtered camera

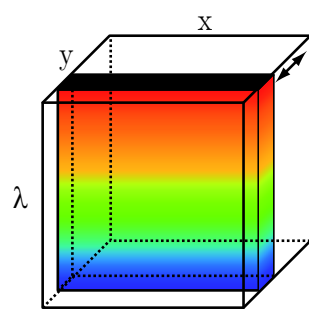

Pushbroom imaging

spectrometer

Figure 2. Spectral imaging sensors classified from the point of view of their data acquisition modes.

an image. In this way, not only spectral mixing is prevented, but also sensitivity to minor components is improved. There still remains the scanning of the other spatial dimension, but this can be easily achieved by the relative motion between the scene and the imaging spectrometer field of view. As a result, this configuration is particularly suitable for industrial applications, which exhibit a predominance of conveyor belt systems.

Since the spectral composition of all objects within the field of view of the instrument is simultaneously measured, hyperspectral imagers allow a much greater percentage of the object to be analysed and provide much higher sampling rates than simple machine vision or single-point spectral instruments. On account of all this, hyperspectral imaging can aid companies in the agri-food and industrial sectors to achieve high speed, in-line inspection and enhanced quality control in their production processes. An overview of approaches based on this technology to address a number of problems in agri-food and industrial sectors is illustrated in this work. A description of these niches is presented in the next lines.

\subsection{Diagnosis and quality control in agri-food industry}

Raw material on-line characterization and discrimination processes are a key issue in many other agricultural ${ }^{5}$ and industrial sectors. ${ }^{6}$ Optical techniques are commonly very suitable for this kind of applications, since they are not only non-destructive but also non-contact. Among them, hyperspectral imaging offers an enormous potential because acquisition systems can be easily installed over the conveyor belt with minimum modifications of the industrial set-up. The problem to be solved here consists in the classification of the raw material at the entrance of a tobacco production plant. Tobacco leaves are intermingled with a great variety of materials such as plastics, cords, cardboard, papers, etc., mainly due to the manual harvest procedure. These spurious elements or "defects" have to be rejected before starting the process of making cigars. At first, the leaves undergo a mechanical classification procedure, where rejection due to material differences of weight, size and density is performed. However, company's suggestions indicate that some of the aforementioned undesired materials go through this coarse classification and additional discrimination systems are required. These procedures are actually performed by time-consuming machine-vision techniques.

\subsection{Arc-welding quality monitoring}

On-line quality monitoring in welding processes is currently an active area of research. Both arc and laser-welding are widely used in some significant industrial scenarios, like aeronautics or nuclear, among others. In some of these situations, where quality plays a major role, the appearance of weld defects has to be prevented, but the lack of an efficient mathematical formulation ${ }^{7}$ increases the complexity of that goal. Procedure trials are commonly employed to solve this problem by establishing the input welding parameters minimizing the occurrence of 


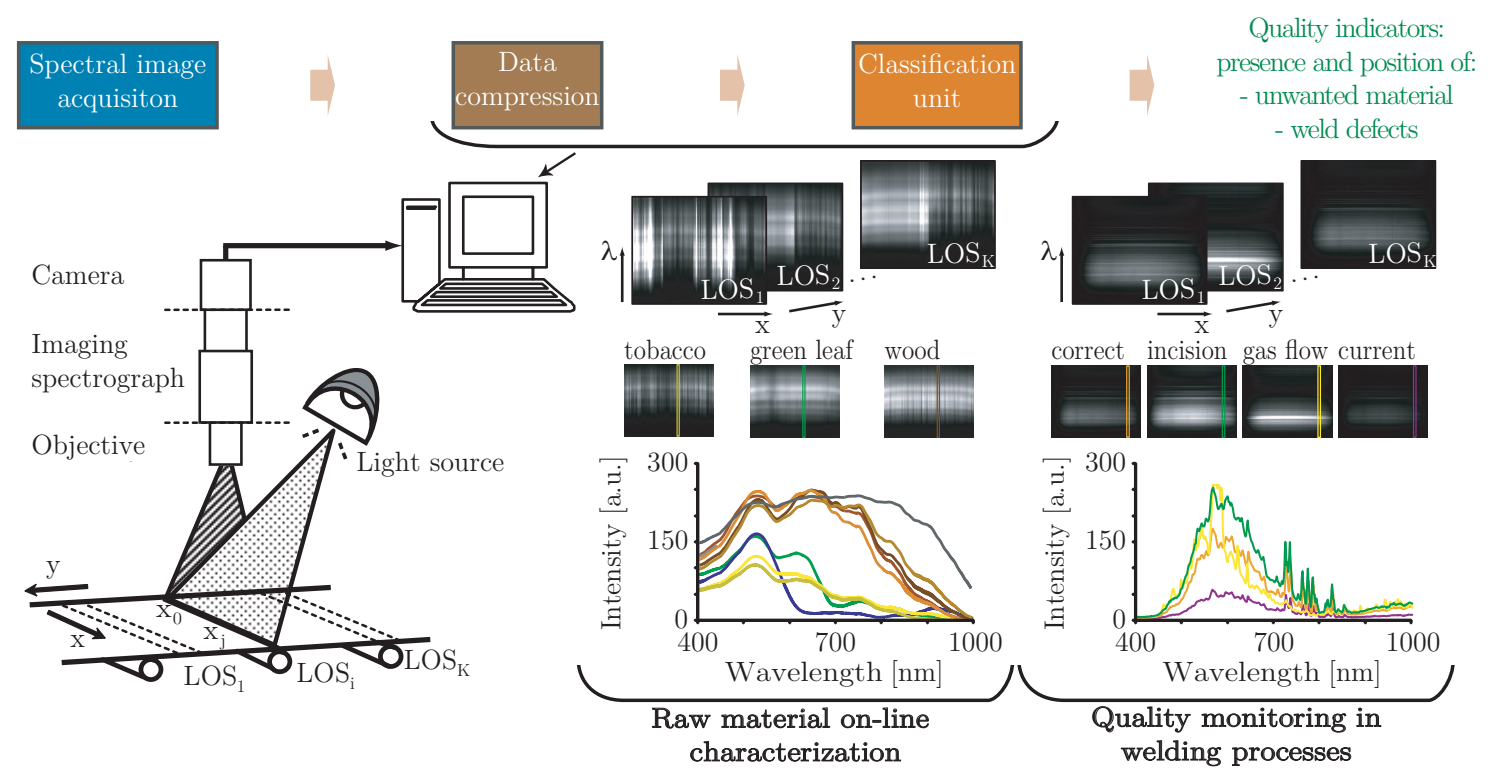

Figure 3. Global block diagram of the designed hyperspectral imaging system for agri-food and industrial applications.

defects. However, different perturbations may appear during the process, thus provoking weld flaws such as porosities, lack of penetration or inclusions. ${ }^{8}$ In this regard, Non-Destructive Evaluation Techniques (NDT) are used off-line, once the process is over, to determine the quality of the seams. X-rays, ultrasounds, penetrant liquids or magnetic particles are examples of these techniques, ${ }^{9}$ which obviously imply a cost, both in economic and productivity terms. The benefits of a robust and reliable welding monitoring system will be, not only the possibility of gaining real-time knowledge regarding the process, but also the opportunity to reduce some of the NDT costs. Plasma optical spectroscopy has proven to be a promising solution, given the immunity of optical fiber based sensors to the strong electromagnetic interference (EMI). Several works have shown that an on-line analysis is feasible, typically by considering simplifications to estimate the plasma electronic temperature $\left(T_{e}\right)$, since this parameter is known to exhibit a direct correlation to the quality of the associated welding. ${ }^{10}$ However, there is still an uncertainty in terms of the plasma lines involved in the analysis, whose intensity is averaged through the line of observation determined by the system input optics. This makes it impossible to carry out a spatial analysis of the plasma column, which could be useful to select the optimum spectral bands in terms of defect discrimination.

\section{HYPERSPECTRAL IMAGING SYSTEM APPROACH}

As stated, hyperspectral systems provide the simultaneous measurement of the light spectra coming from all the points along the line of sight over the object surface. From this huge amount of information, a usable approach should supply an output about material characteristics or composition. With this aim, the system block diagram depicted in Figure 3 has been envisaged. Optical parameters are firstly measured as a function of wavelength and spatial position. The reduction of the data volume collected by hyperspectral imagers is carried out in the data compression block and, finally, quality indicators and figures of merit (e.g. position of the "defects" to determine which jets of pressurized air need to be activated to blow the unwanted material into a container) are obtained in the classification unit. 


\subsection{Spectral image acquisition}

Hyperspectral images of the raw material or the plasma column are recorded through a PGP (Prism - Grating Prism) imaging spectrograph, ${ }^{11}$ which is based on a pushbroom principle, connected between an objective lens and a monochrome camera. The equipment is known as ImSpector and it is commercialised by Specim Ltd. As an example, some hyperspectral images are also depicted in Fig. 3. The optical spectrum components of the diffuse reflectance (raw material) or the atomic emission (welding) are measured simultaneously for every point $x_{i}$ in the observation line (LOS, Line Of Sight) and the movement of the conveyor belt or the welding torch automatically forms the other spatial dimension. A light source, e.g. a halogen lamp with a quasi-flat spectrum, is usually required to illuminate the raw materials to be measured. In the welding case this is not necessary because the spectrum of the radiation emitted by the plasma is directly acquired.

The image data of the raw material samples were measured through two different hyperspectral systems, working respectively in the Vis-NIR and NIR ranges of the spectrum. Accordingly, versions V10E and N24E, whose spectral ranges cover from 400 to $1000 \mathrm{~nm}$ and from 1000 to $2400 \mathrm{~nm}$ were employed. Illumination consists, in both cases, of two halogen floodlights Tasley MX500, with a power rating of 500W each. Selected C-mount lens are respectively the Navitar objective lens Zoom 7000 and the Pentax C1614-M (C31634). Finally, the sensor module includes in the Vis-NIR range a high-performance monochrome digital camera, the Pixelink PL-A741 (1280 x 1204 pixels) whose spectral range goes from 350 up to $1000 \mathrm{~nm}$. In the NIR system the monochrome camera XEVA-FPA-2.5-320 (320 x 256 pixels) by Xenics, whose spectral range is from 850 to $2500 \mathrm{~nm}$, has been employed. Both systems are controlled by a desktop PC (Pentium IV with a 3 GHz processor and RAM-512 MB). Image processing tasks are performed with Matlab. ${ }^{12}$ Communication interface between computer and cameras is IEEE1394 for the Pixelink PL-A741 and Camera Link for image transference and USB for camera control in the case of the XEVA-FPA-2.5-230.

Hyperspectral images of the plasma column were only recorded through the acquisition system working in the Vis-NIR range, where the lens focusing range was manually controlled to cover the entire width of the plasma column.

\subsection{Relevant information extraction from hyperspectral images}

The result of acquisition are digital images as those shown in Fig. 3, where each column represents the discrete spectrum values of a spatial position within the field of view of the corresponding system. The entire wavelength range is acquired by hyperspectral systems and, as highlighted more clearly in the average spectral curves depicted at the bottom of Fig. 3, some ranges of the spectra exhibit similar characteristics. This indicates that these spectral bands will provide the same information to discriminate spurious elements from wanted material or defects from correct welds. Consequently, data volume reduction techniques are employed in the Data compression block, which carries out the compression of both, spectral and spatial, axes of the captured image. The spatial axis is simply averaged, i.e. the mean spectrum of every five adjacent pixels is calculated. The aim is to reduce the noise present in the measurements. There are a wide variety of characterization methods ${ }^{13}$ to obtain the relevant information, i.e. fingerprint, from the spectral curves and they achieve this objective essentially by two different ways. Feature extraction methods lie on the projection of the spectral data to a lower dimension space. On the other hand, feature selection methods identify and ignore those bands that do not contribute to the classification task and they have several advantages such as the interpretability of results, i.e. the selected features are spectral bands with physical meaning and results are extrapolated to other spectrometers with different spectral bands.

Proc. of SPIE Vol. 7726 77260E-5 
In this stage, the main risk is the loss of information if the feature selection is wrong. Different versions of Principal Component Analysis (PCA) ${ }^{14-16}$ as well as a feature selection method based on a Sequential Floating Feature Selection (SFFS) ${ }^{17,18}$ algorithm, are considered to implement the data compression unit of the system. PCA obtains the vectors or projection directions by maximizing the projected variance of a given data set. For dimensionality reduction purposes, the number of projection directions should be truncated trying to lose as less information as possible. The conventional application of PCA (the so-called "m-method") selects and filters the vectors in terms of their eigenvalues. The more advanced versions of PCA select the projections that best separate the mean of the individual classes ("J-measure"), considering for this both means and variances of the classes ("SEPCOR"). Another option is modifying the original data set to include information related with the data class (SPCA, Supervised Principal Component Analysis).

\subsection{Hyperspectral image discrimination}

Although compressed hyperspectral images still contain intensive information about the optical properties of the objects only the presence and position of spurious elements or weld flaws are of interest. This segmentation of the images in areas that exhibit the same spectral behavior is accomplished in the last stage, i.e. in the Classification unit. New spectral interpretation algorithms, new variants of existing algorithms, and new implementations of existing methods appear all the time. ${ }^{19}$ Since the large number of papers published in attempts to establish the relative superiority of any of these algorithms have failed, any of them could be employed for the identification task. Artificial Neural Networks (ANN), ${ }^{20}$ because of their ability to handle non-linearity, their parallel processing of information and their quick adaptability to system dynamics, ${ }^{21}$ were initially employed in material discrimination. ANNs proved, with the appropriate training, very accurate in spurious material detection. ${ }^{22}$ This suggested that possibly simpler algorithms, where simple means that they provide linear solutions, could be also employed. Classification units based on Fisher's Discriminant Analysis $(\mathrm{FLD})^{23,24}$ and Spectral Angle Mapper (SAM $)^{25,26}$ have been proposed too. Their discrimination capability and time performances will be shown and compared in following sections. The application of any of these algorithms to hyperspectral image interpretation involves two phases: training and test stages. At first, the classifier parameters are determined, for example in the case of ANNs, the number of hidden layers and nodes per layer, training algorithms, etc. are adjusted in order to produce outputs that match the previously known results for the training data set. In the test phase, classification outputs are provided for unknown inputs based on the parameters calculated in the previous stage. Training phases are though configured with homogeneous samples as test samples, which means, for instance, in material discrimination that all the pixels across the observation line corresponded to the same compound. For the test phase, different materials were combined in the same line to confirm that the system was able to spatially discriminate between them. This two-step approach has a main practical limitation. When a new and unknown material needs to be classified, it is compulsory to interrupt system operation and calibrate it again. This could be avoided by an implementation of the discrimination unit based on the $\mathrm{K}$ nearest neighbour (KNN) algorithm. ${ }^{27}$ No training is required, because the classification of a new material is performed by means of a comparison with the whole set of previously known materials.

Only the expected performance of supervised classification algorithms has been estimated. Unsupervised algorithms normally have worse performances because they do not take into account class distributions and they only form clusters as a function of data similarity. But this similarity could be only related to spectrum features and not to the discriminant information among the classes. Unsupervised classification algorithms are traditionally employed in remote sensing to deal with the typically few and costly labeled data, but since this 
problematic issue does not occur in the applications in agri-food and industrial environments, they will not be employed in the implementation of the classification unit.

\section{RAW MATERIAL DISCRIMINATION}

As mentioned in the Introduction, among the different industrial manufacturing processes where raw material discrimination and classification at the input chain is a key quality control stage (e.g. food, beverage, etc.), tobacco industry has been selected as the application case. It was selected, first of all, because samples could be provided by a collaborative partner in a R\&D project, Altadis S.A., which is a multinational purveyor and manufacturer of cigarettes, tobacco and cigars. Apart from company interest in tobacco sorting, there are a few studies about the spectral characterization of its chemical components ${ }^{30}$ and even less is the reported work in open literature regarding its quality control in the industry, which is, as mentioned above, performed by timeconsuming machine vision techniques. Additionally, the developed strategies of classification could be extended to other sorting problems.

In order to distinguish the spurious materials intermingled with the leaves, the classification stage considered two different classes:

- target: tobacco leaves.

- non-target: undesired materials appearing with tobacco due to the manual harvest procedure. This class includes wood, cardboard, different colored cellophanes, leather, foil, paper of sweets, textile threads and brown and green leaves of other vegetable material different from tobacco. A priori, vegetable species could share some morphology and spectral characteristics with tobacco leaves but they must be discriminated from them. On account of this, vegetable material different from tobacco will be referred from now on as "closest-non-target".

Figure 4 shows the clustering degree of the classes, "target" and "non-target", when they are projected as a function of the first three principal components (feature extraction alternatives) or wavelengths (feature extraction) selected by each considered method, i.e. after their processing in the data compression unit of the system.

All the methods behave reasonably well in the clusterization of tobacco leaves, but the spreading of the "non-target" class is significant. This is obviously explained due to the clear different morphology and spectral behavior of the non-desired materials. Attending to Fig. 4 it could be concluded that a posterior classification unit is required to performed material discrimination. Figure 5 shows a comparison between aforementioned discrimination methodologies from a qualitative point of view. A thorough and comprehensive work programme that included a wide variety of test samples was performed in this last stage. Figure 5 illustrates a couple of samples and their corresponding results. Its horizontal lines contain the following information:

- A photograph of the sample under analysis. The white lines delimit the observation line, in such a way that the horizontal axis corresponds to the spatial axis of the spectrograph.

- A zoom of the scene line. 

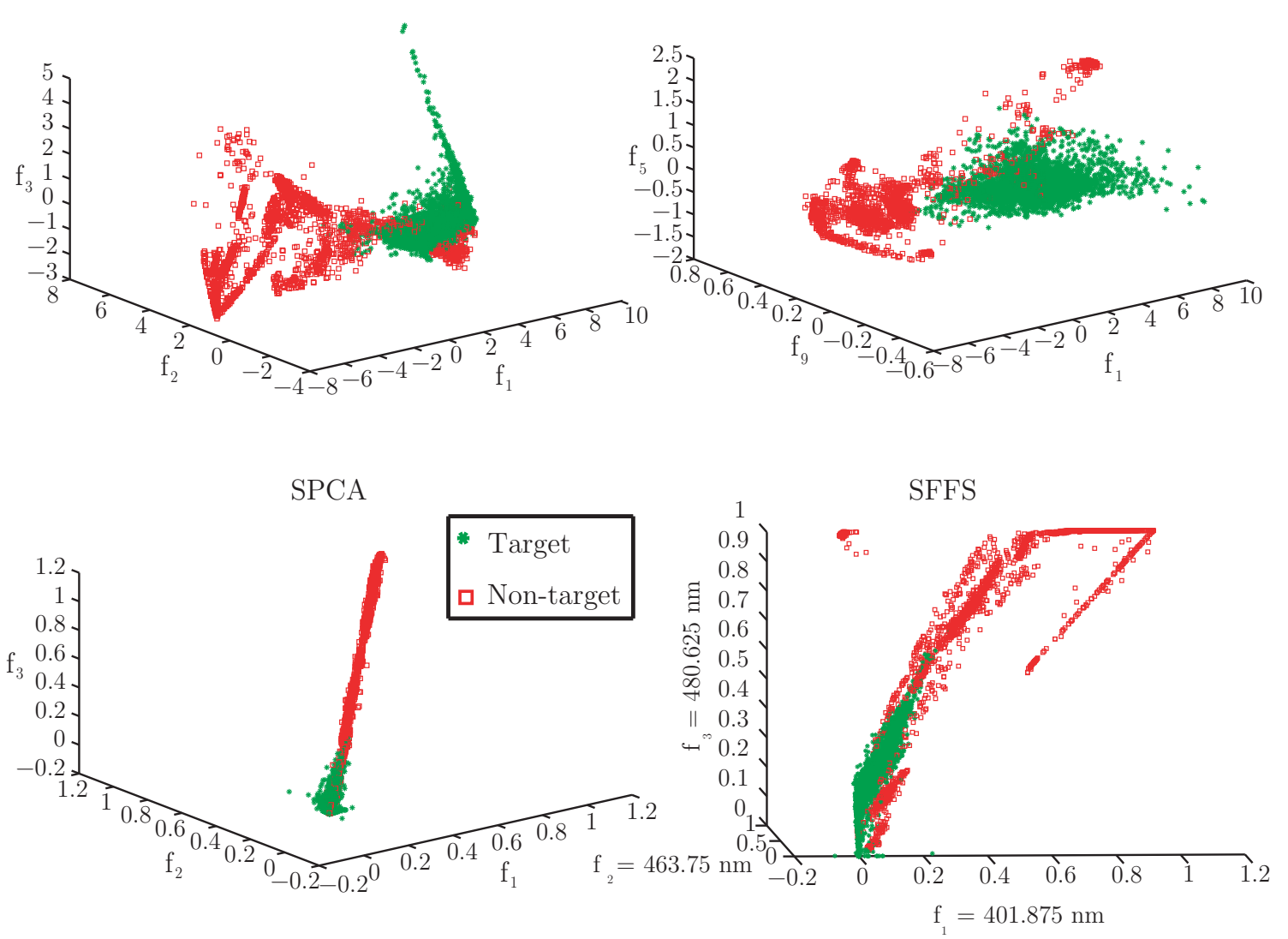

Figure 4. Grouped scatter plots of material spectra distinguishing the wanted raw material ("target") and spurious elements.

- The spectral images. As before, the spatial axis is the horizontal with the origin on the left as it appears in the real photograph. The spectral axis is the vertical one with the smallest wavelength of the spectral range at the bottom of the image.

- The results of the classification provided by the neural network when data dimensionality reduction is based on PCA (m-method).

- Idem but data compression is performed in this case by means of SFFS.

- Classification provided by the linear discriminant. The projection on the discriminant function of the reflectance of each spatial pixel of the observation line is also plotted in black. The decision threshold is plotted in green, showing those pixels of the observation line where the projection is higher than the threshold. These localizations are classified as "target" material.

- Last line shows the SAM outputs.

Test samples shown in Fig. 5 suggest that ANNs provide best classification performance than FLD and SAM. In fact, if a 3-fold cross validation procedure (employing 11520 spectra from 120 images, 80 images of "target" material and 40 of "non-target" material) is followed, ANNs give an average accuracy of $99.56 \%$, a success 


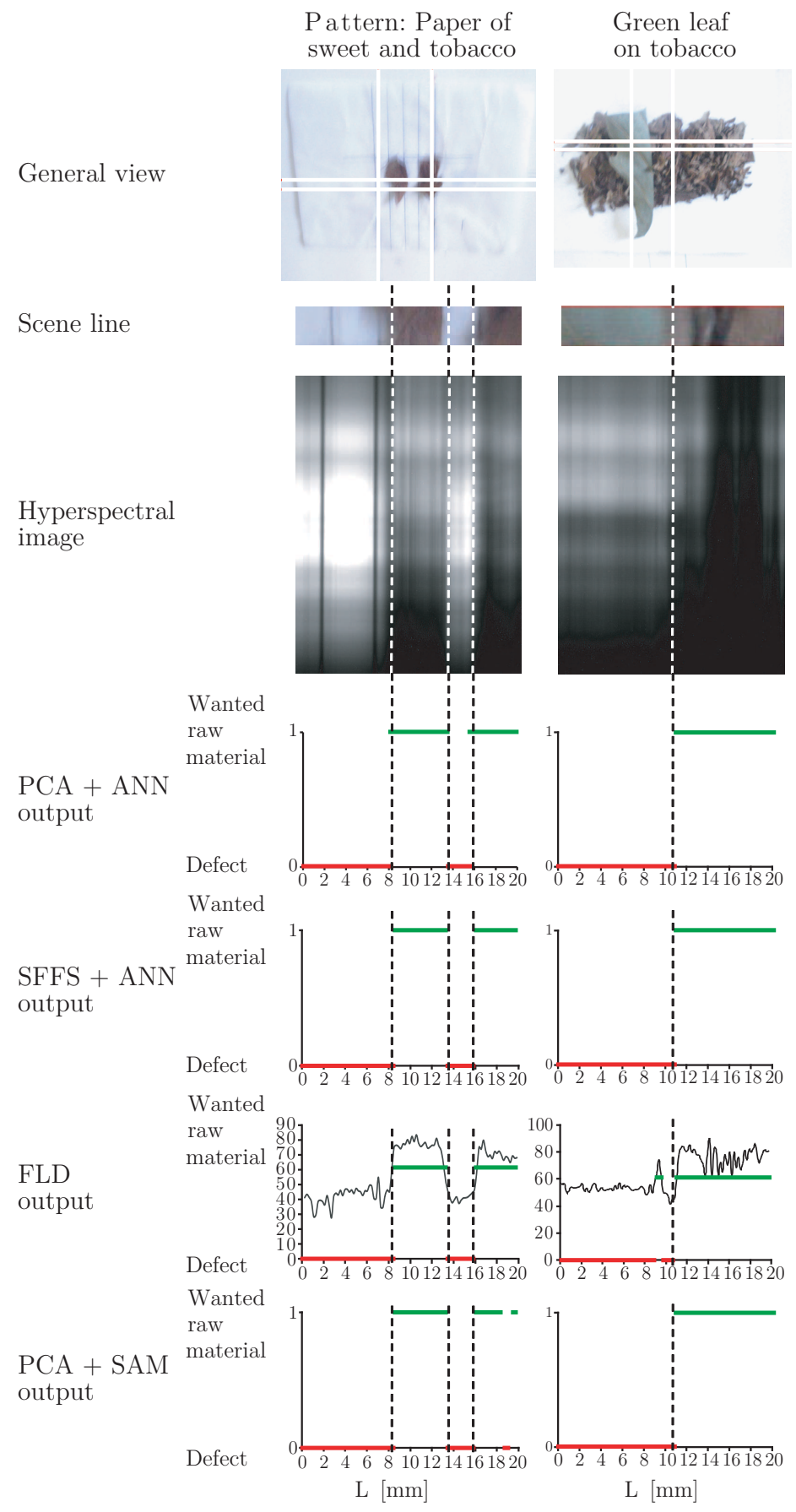

Figure 5. Grouped scatter plots of material spectra distinguishing the wanted raw material ("target") and spurious elements. 


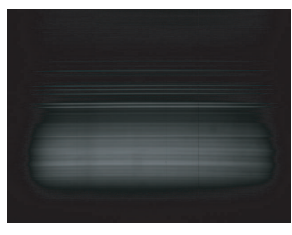

a)

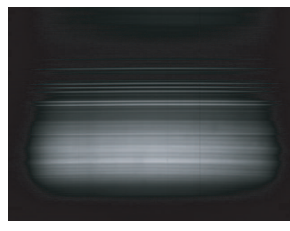

b)

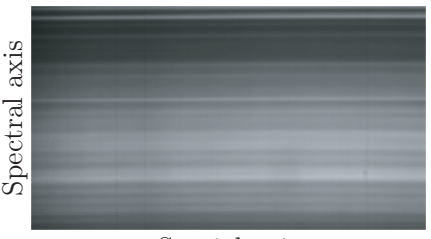

Spatial axis

c)

Figure 6. a) Hyperspectral image of the plasma column for a seam performed with 26 A. b) Hyperspectral image of the plasma column for a seam performed with 50 A. c) Spectral capture of a seam performed with 50 A.

probability of $92.87 \%$ was achieved with FLD and SAM provided an accuracy of $90.54 \%$. However, this does not imply that ANNs are better, they only have higher classification performance. FLD and SAM have, due to their linearity and simplicity, better time performance, so if figure errors are acceptable, they would be preferable. This exact situation is the actual one also in remote sensing, it has not been encountered yet, as pointed by Manolakis et al. ${ }^{19}$ the best hyperspectral detection algorithms. It depends on the particular requirement applications.

\section{ARC-WELDING QUALITY ASSURANCE}

A great variety of weld defects arise from an insufficient welding current. As a consequence, the first aim is to demonstrate the feasibility of hyperspectral imaging techniques to identify this issue. Two examples of seams performed with constant welding currents of 26 and $40 \mathrm{~A}$ are presented in Figure $6 \mathrm{a}$ ) and b). A detail of the plasma column can be seen in Fig. 6 c), where several plasma emission lines are to be found. Although different approaches could be considered, it does not seem necessary with this solution to perform an identification of these plasma emission lines, i.e. the association of each emission line with its corresponding chemical species, in terms of the ability of the system to detect weld defects. In this point it should be noted that, initially, the main purpose was to elucidate whether this kind of solution could be used in that regard. Given the kind of images acquired by the system, it seems that a simple image processing approach would be more efficient; apart from the lack of optical resolution (in the order of $2.8 \mathrm{~nm}$ ), which would make it difficult to perform a reliable line identification. ${ }^{31}$

Another good example to illustrate the performance of HSI approach in welding quality is exhibited in Figure 7, where a seam was performed with a constant welding current of $37 \mathrm{~A}$ and a protection gas flow rate of 12 $\mathrm{L} / \mathrm{min}$. This rate was manually altered to simulate a perturbation on the gas flow, reducing it to approximately $2 \mathrm{~L} / \mathrm{min}$ during $0.5 \mathrm{~s}$. This reduction in the protection gas flow is also a common cause of weld defects. A sequence of the images acquired by the hyperspectral imaging system is presented in Fig. 7, where Figs. 7 a), c) and e) are associated with the correct welding conditions, and Figs. 7 b) and d) with the simulated defects. In this particular case the differences between the captured images are obvious, and, consequently, the defects could be easily identified. Figure $7 \mathrm{f}$ ) shows a comparison with traditional plasma electronic temperature profiles employed in seam flaw detection, where the two gas shortages can be clearly identified.

Finally, the use of imaging spectroscopy applied to on-line welding quality monitoring was explored and, to the best of the author knowledge, there is no employment of hyperspectral techniques to image the plasma column of welding processes previously reported. In comparison to the traditional spectroscopic approach, the use of hyperspectral devices allows to capture detailed images of the plasma column with both spectral and spatial information. However, a more extensive study with industrial samples should be still conducted, including 


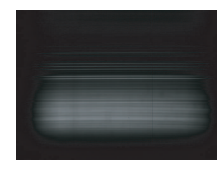

a)

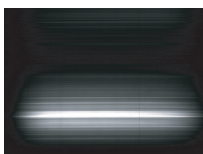

b)

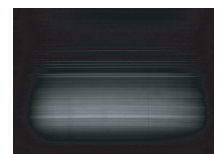

c)

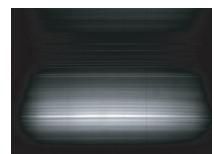

d)

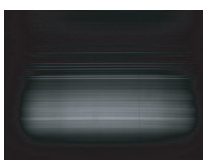

e)

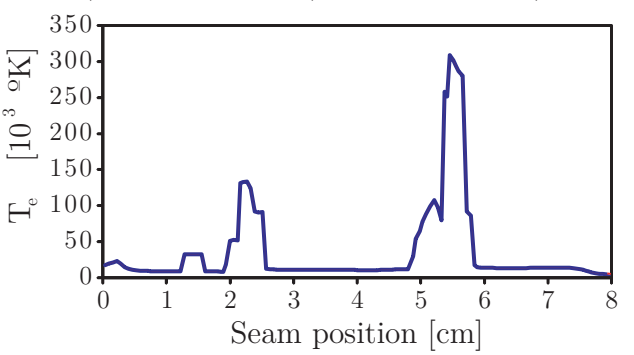

f)

Figure 7. a), c) and e) Hyperspectral images of the plasma column for a seam performed with 37 A and gas flow rate 12 L/min. b), d) Gas flow rate reduction. f) Plasma electronic temperature profile for the defective seam.

different materials, shielding gasses, and flaws, since these are only preliminary studies on the feasibility of the approach.

\section{CONCLUSIONS}

The enormous potential of hyperspectral imaging spectroscopy for agri-food and industrial applications has been demonstrated throughout this work. It shows interesting potential to replace analytical instrumentation more expensive and cumbersome, in order to achieve a quick quality control and product classification. Field tests of a preliminary version of the sensor proposed for welding quality monitoring is currently under evaluation to detect the appearance of defects in arc-welding-tube-tubesheet process of steam generators for nuclear plants. Although a validation of the system for tobacco raw material discrimination was designed, it has not been tested yet in a real industrial scenario. Tobacco consumption is decreasing due to recent anti-tobacco legislations, and, as a consequence, the interest of tobacco companies in the adoption of novel technologies has declined. However, the underlying imaging spectroscopy technique can be easily extended to other industrial sectors where it can demonstrate its enormous potential. A study on the extendibility of the system to improve the economical performance of a textile industry is ongoing.

\section{ACKNOWLEDGMENTS}

This work has been co-supported by the project TEC2007-67987-C02-01. Authors also want to thank J.J. Valdiande and F. Anabitarte for their help and support during the experimental tests.

\section{REFERENCES}

1. W. Schmidt, Optical spectroscopy in chemistry and life sciences, Wiley-VCH, 2005.

2. S. J. Millar, M. B. Whitworth, A. Chau, and J. R. Gilchrist, "Mapping food composition using nir hyperspectral imaging," New Food 3, pp. 34-9, 2008.

3. C. Willoughby, M. Folkman, and M. Figueroa, "Application of hyperspectral imaging spectrometer system to industrial inspection," Proc. of SPIE 2599, pp. 264-72, 1996. 
4. O. Conde, A. Cobo, P. Cantero, D. Conde, J. Mirapeix, A. Cubillas, and J. Lopez-Higuera, "Adaptive illumination source for multispectral vision system applied to material discrimination," Proc. of SPIE 7000, pp. 70000Z1-11, 2008.

5. J. Xing, C. Bravo, P. Jancsk, H. Ramon, and J. Baerdemaeker, "Detecting bruises on Golden Delicious apples using hyperspectral imaging with multiple wavebands," Biosystems Engineering 90 (1), pp. 27-36, 2005.

6. E. Herrala, T. Hyvarinen, O. Voutilainen, and J. Lammasniemi, "An optoelectronic sensor system for industrial multipoint and imaging spectrometry," Sensor. Actuat. A-Phys. 61 (1-3), pp. 335-8, 1997.

7. C. Wu and M. Ushio, "Analysis of the TIG welding arc behaviour," Comp. Mater. Sci. 7 (3), pp. 308-14, 1997.

8. A. Ancona, P. Lugara, F. Ottonelli, and I. Catalano, "A sensing torch for the on-line monitoring of the gas tungsten arc welding process of steel pipes," Meas. Sci. Technol. 15 (12), pp. 2412-8, 2004.

9. A. S. for Nondestructive Testing ASNT, Nondestructive handbook on Infrared Technology Vol. 3: Infrared and thermal testing, ASNT Press, 2001.

10. A. Ancona, V. Spagnolo, P. Lugara, and M. Ferrara, "Optical sensor for real-time monitoring of $\mathrm{CO}_{2}$ laser welding process," App. Opt. 40, pp. 6019-25, 2001.

11. T. Vaarala, A. Aikio, and H. Keraenen, "An advanced prism-grating-prism imaging spectrograph in on-line industrial applications," Proc. of SPIE 3101, pp. 322-30, 1997.

12. T. M. Inc., "Matlab reference manual.".

13. J. Workman and A. W. Springsteen, Applied spectroscopy: a compact reference for practitioners, Academic Press, 1998.

14. T. Moeslund, "Principal component analysis. An introduction," tech. rep., Aalborg University CVMT 01-02.

15. Y. Koren, Q. Tian, X. Zhou, and T. Huang, "Robust linear dimensionality reduction," IEEE T. Vis. Comput. Gr. 10 (4), pp. 459-70, 2004.

16. M. Park and J. Choi, "Theoretical analysis on feature extraction capability of class-augmented PCA," Pattern Recogn. 42, pp. 2353-62, 2009.

17. F. Ferri, P. Pudil, M. Hatef, and J. Kittler, Comparative study of techniques for large-scale feature selection in Pattern Recognition in Practice IV: Multiple Paradigms, Comparative Studies and Hybrid Systems, Amsterdam: North-Holland Publishing Company, 1994.

18. L. Gomez-Chova, L. Calpe, G. Camps-Valls, J. D. Martin, E. Soria, J. Vila, L. Alonso-Corda, and J. Moreno, "Feature selection of hyperspectral data through local correlation and SFFS for crop classification," IEEE Int. Geosci. Remote Sens. Symp. Proc. 1, pp. 555-7, 2003.

19. D. Manolakis, R. Lockwood, T. Cooley, and J. Jacobson, "Is there a best hyperspectral detection algorithm?," Proc. of SPIE 7334, p. 733402, 2009.

20. C. Chen, Fuzzy logic and neural network handbook, McGraw Hill, 1996.

21. A. Nogueira, M. de Oliveira, P. Salvador, R. Valadas, and A. Pacheco, "Classification of internet users using discriminant analysis and neural networks," in NGI 2005: Next Generation Internet Networks: Traffic Engineering, pp. 341-8, 2005.

22. P. Garcia-Allende, O. Conde, A. Cubillas, C. Jauregui, and J. Lopez-Higuera, "New raw material discrimination system based on a spatial optical spectroscopy technique," Sensor. Actuat. A-Phys. 135 (2), pp. 605-12, 2007. 
23. D. Michie, D. Spiegelhalter, and C. Taylor, Machine learning, neural and statistical classification, Prentice Hall, 1994.

24. P. Garcia-Allende, O. Conde, J. Mirapeix, A. Cobo, and J. Lopez-Higuera, "Quality control of industrial processes by combining a hyperspectral sensor and Fisher's linear discriminant analysis," Sensor. Actuat. B-Chem. 129 (2), pp. 977-84, 2008.

25. G. Girouard, A. Bannari, A. E. Harti, and A. Desrochers, "Validated spectral angle mapper algorithm for geological mapping: comparative study between Quickbird and Landsat-TM," in Geoimagery bridging continents 2004, pp. 599-604, 2004.

26. P. Garcia-Allende, O. Conde, J. Mirapeix, A. Cubillas, and J. Lopez-Higuera, "Data processing method applying principal component analysis and spectral angle mapper for imaging spectroscopic sensors," IEEE Sens. J. 8 (7), pp. 1310-6, 2008.

27. K. Fukunaga, Introduction to statistical pattern recogniton, Academic Press, 1990.

28. H. Zhu and R. Rohwer, "No free lunch for cross-validation," Neural Comput. 8 (7), pp. 1421-6, 1996.

29. C. Goutte, "Note on free lunches and cross validation," Neural Comput. 9 (6), pp. 1245-9, 1997.

30. W. McClure, K. Horris, and W. Weeks, "Rapid spectrophotometric analysis of the chemical composition of tobacco, Part 1: Total reducing sugars," Beitrge zur Tabakforschung International 9 (1), pp. 13-8, 1997.

31. J. Mirapeix, A. Cobo, O. Conde, C. Jauregui, and J. Lopez-Higuera, "Fast algorithm for spectral processing with application to on-line welding quality assurance," Meas. Sci. Technol. 17 (10), pp. 2623-9, 2006. 